\title{
Deposition of Magnetite Nanoparticles in Activated Carbons and Preparation of Magnetic Activated Carbons
}

\author{
S. A. Kahani, M. Hamadanian and O. Vandadi \\ Department of Chemistry, University of Kashan, 87317-51167 Kashan, Iran
}

\begin{abstract}
Magnetic activated carbons (MACs) for gold recovery from alkaline cyanide solutions have been developed by mixing a magnetic precursor with a carbon source, and treating the mixture under controlled conditions. As would be expected, these activated carbons have high specific surface areas due to their microporous structure. In addition, the small particle size of the MACs produced allows rapid adsorption of gold in solution, and the magnetic character of these MACs enables recovery from suspension by magnetic separation.
\end{abstract}

Keywords: Magnetite; Magnetic activated carbons; gold recovery.

PACS: 75.47.Lx; 75.75+a; 81.07-b

\section{INTRODUCTION}

Magnetic separation has been developed as a recovery and pollution-control process for many environmental and industrial problems. Application of efficient magnetic filtration to petroleum-related decontamination and waste treatment operations is attractive because it can provide rapid removal of contaminants from aqueous waste streams [1]. The simplest method was to mix an extremely large excess of magnetite oil (fatty acid) by weight so that the oil absorbed onto the surface of the magnetic powder. Activated carbon has a long-standing history as an absorbent and extraction agent of Precious metals such as gold from its ore [2-3]. Thus, conversion of activated carbon into a magnetically-active material would provide an excellent magnetic filtration aid. Here a method proposed for the preparation of porous carbon that was loaded with magnetite particles. Magnetic activated carbons (MACs) for gold recovery from alkaline cyanide solutions have been developed by mixing a magnetic precursor with a carbon source, and treating the mixture under controlled conditions. As would be expected, these activated carbons have high specific surface areas due to their microporous structure. In addition, the small particle size of the MACs produced allows rapid adsorption of gold in solution, and the magnetic character of these MACs enables recovery from suspension by magnetic separation. Magnetic carbon active applied as magnetic carriers that could be used in the recovery of gold from cyanide solutions. Based on their results, in this investigation the extraction of gold up to $99 \%$ can be achieved.

\section{EXPERIMENTAL}

Reagents used were of analytical grade and were obtained from Merck Co. All the preparations were carried out under the nitrogen and solvents were of technical grade, used after distillation. XRD measurements were performed using a Philips X,pert pero MPD diffractometer with $\mathrm{Cu} \mathrm{K \alpha}$ radiation in the range $2 \theta$ from 10 to 80 , at room temperature. IR was obtained as $\mathrm{KBr}$ pellet in the range 4000 to $500 \mathrm{~cm}^{-1}$ using Shimadzu FT-IR spectrometer. The vibrating sample magnetization (PAR-VSM155R) was used to evaluate the magnetic parameters. Scanning electron micrographs (SEM) were recorded, without sample coating, by JEOL JSM-5600LV.

CP929, Nanotechnology and Its Applications, First Sharjah International Conference edited by Y. I. Salamin, N. M. Hamdan, H. Al-Awadhi, N. M. Jisrawi, and N. Tabet (C) 2007 American Institute of Physics 978-0-7354-0439-7/07/\$23.00 


\section{Preparation of Magnetite Using a Homogenous Method}

The solutions containing $2.72 \mathrm{~g}$ of $\mathrm{FeCl}_{3}$ and $1 \mathrm{~g}$ of $\mathrm{FeCl}_{2}$ were mixed at a certain molar ratio. Then $20 \mathrm{~mL}$ of $\mathrm{NH}_{4} \mathrm{OH} 25 \%$ was slowly injected into the mixture of $\mathrm{FeCl}_{3}$ and $\mathrm{FeCl}_{2}$ under vigorous stirring at temperature $60-70{ }^{0} \mathrm{C}$. Before the reaction, $\mathrm{N}_{2}$ gas was injected through the reaction medium to prevent possible oxidation. After precipitation, the $\mathrm{Fe}_{3} \mathrm{O}_{4}$ particles were repeatedly washed and filtered before drying at room temperature [4]. The chemical reaction of $\mathrm{Fe}_{3} \mathrm{O}_{4}$ precipitation can be described as follows

$$
\mathrm{Fe}^{2+}+2 \mathrm{Fe}^{3+}+8 \mathrm{OH}^{-} \rightarrow \mathrm{Fe}_{3} \mathrm{O}_{4}+4 \mathrm{H}_{2} \mathrm{O}
$$

\section{Doping of Magnetite in Activated Carbon}

A solution containing $2 \mathrm{~g}$ of magnetite and $2 \mathrm{~g}$ of commercial activated carbon was prepared in $40 \mathrm{ml}$ distilled water under vigorous stirring at room temperature. After 5 hours the doping is complete. Filtering and washing and drying in an oven at $100{ }^{\circ} \mathrm{C}$ are carried out.

\section{Preparation of Magnetite Using a Heterogeneous Method}

A mass of 8.9 gram $(0.1$ mole $)$ of goethite, 2 g activated carbon and $9.94 \mathrm{~g}(0.05$ mole $) \mathrm{FeCl}_{2} .4 \mathrm{H}_{2} \mathrm{O}$ in $250 \mathrm{~mL}$ deionized water are placed in a 500-mL round-bottom flask with a side arm gas inlet tube (flushed with $\mathrm{N}_{2}$ ) and a reflux condenser and a magnetic stirring bar are placed in the flask. This is followed by adding $50 \mathrm{~mL}$ of $2 \mathrm{M} \mathrm{NaOH}$ with vigorous stirring. The reaction mixture is heated to the boiling point, and reflux is maintained for 1-2 hours. The reaction should be carried out under $\mathrm{N}_{2}$. During the transformation the $\mathrm{pH}$ falls to 8-9 and a black precipitate is formed in activated carbon. After the reaction is complete the product was repeatedly washed with distilled water and filtered and dried in an oven at the temperature $100{ }^{\circ} \mathrm{C}$. The chemical reaction of $\mathrm{Fe}_{3} \mathrm{O}_{4}$ precipitation can be described as follows

$$
\mathrm{Fe}^{2+}+2 \mathrm{FeOOH}+2 \mathrm{OH}^{-} \rightarrow \mathrm{Fe}_{3} \mathrm{O}_{4}+2 \mathrm{H}_{2} \mathrm{O}
$$

\section{Gold Adsorption on MACs}

A 1-liter solution containing $10 \mathrm{mg}$ of gold as the aurodicyanide complex $\mathrm{Au}(\mathrm{CN})_{2}$ in 1 liter of solution $(10 \mathrm{ppm} \mathrm{Au})$ at $\mathrm{pH} 11$, a free sodium cyanide concentration of $0.02 \mathrm{M}$, and at room temperature $\left(25^{\circ} \mathrm{C}\right)$ was stirred at $1500 \mathrm{RPM}$ with an overhead motor. One gram of magnetic activated carbon was added to the system. Samples of $10 \mathrm{~mL}$ were taken at specific times with a syringe, the solution was filtered, and the amount of gold in solution was determined by AAS. Based on the amount of gold in solution after specific adsorption times, the $\%$ of gold adsorption at each time could be calculated.

\section{RESULTS AND DISCUSSION}

Magnetic particles have shown great promise as a base material for engineering carriers in separation science and technology. Magnetic particles used in these areas are called magnetic carriers [5]. The objective of magnetic carrier technology is to confer the magnetic property to a naturally nonmagnetic target so that the target can be separated from the stream using magnetic separators. This is particularly important for separations in a complex multiphase system. To make a target magnetic, the target has to be recognized by and attached to a magnetic carrier. This requires the magnetic particles to be usually on a surface or produced in situ formation of a magnetic core in a structured template such as active carbon, porous silica, or polymer. The formation of magnetic cores in porous media is important in magnetic separation. Examples have been given for magnetic carriers of tailored functionalities in a variety of applications, including industrial effluent detoxification, metal recovery, biological cell separation, drug delivery, and the preparation 
of biological sensors. Activated carbon was introduced as an adsorbent for the recovery of precious metals such as gold and silver from cyanide solutions. In recent years activated carbon recovery systems have gained very wide acceptance in the gold industry. Here we proposed the doping of magnetic particles in activated carbon. Magnetic activated carbon applied as a magnetic carrier that could be used in the recovery of gold from cyanide solutions. Activated carbon is a highly porous material with very large intra-particulate area per unit of mass. This requires the magnetic particles for preparation of Magnetic activated carbon to be extremely small, in the nanometer range. The magnetite particles and Magnetic activated carbon prepared are characterized by Fourier transform infrared spectroscopy (FTIR), X-ray powder diffraction (XRD), vibrating sample magnetometry (VSM) and scanning electron microscopy (SEM) and X-Ray Microanalysis.

\section{Infrared Spectroscopy}

The most widely applied techniques for characterizing of iron oxide involve infrared. For iron oxide, IR spectroscopy is useful as a means of identification. This technique also provides information about crystal morphology, and hence the nature of surface hydroxyl groups and adsorbed water. The IR spectra of various magnetite samples show broad bands at 580 and $400 \mathrm{~cm}^{-1}$. The IR spectrum of magnetic activated carbon $\left(\mathrm{MAC}_{1}\right)$ shows broad bands at 1185, 598 and $401 \mathrm{~cm}^{-1}$. The intense band in the region $1000-1185 \mathrm{~cm}^{-1}$ can be attributed to stretching of $\mathrm{C}-\mathrm{C}$ and $\mathrm{C}-\mathrm{O}$ in activated carbon and 581 and $401 \mathrm{~cm}^{-1}$ can be attributed to deposited magnetite. Magnetic activated carbon $\left(\mathrm{MAC}_{1}\right)$ shows broad bands in the region 1000-11200, 614 and $420 \mathrm{~cm}^{-1}$. The intense bands at $1000-1200 \mathrm{~cm}^{-1}$ can be attributed to stretching of C-C and C-O in activated carbon and 614 and $420 \mathrm{~cm}^{-1}$ can be attributed to F-O stretching deposited magnetite. Infrared spectroscopy demonstrated the presence of magnetite in activated carbon phase.

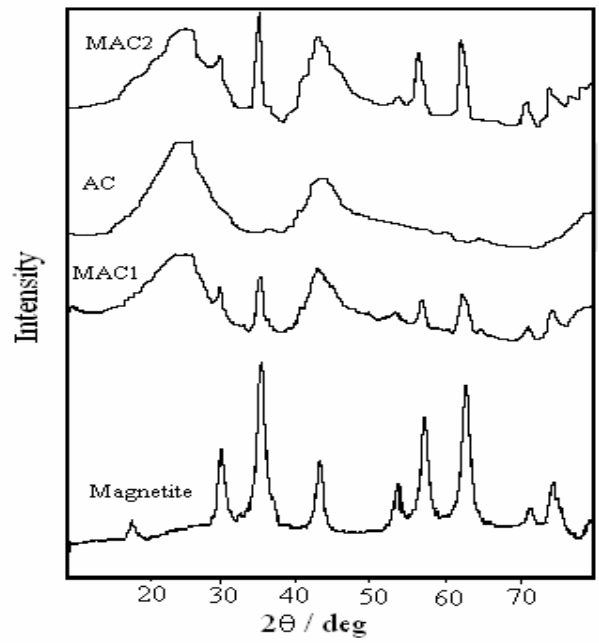

FIGURE 1. X-ray diffraction pattern of magnetite, $\mathrm{MAC}_{1}, \mathrm{AC}$ and $\mathrm{MAC}_{2}$.

\section{X-ray Powder Diffraction}

One of the best methods in characterization of these products is powder X-ray diffraction. A few years after the first successful application of X-ray diffraction to crystal structure determination, this technique was used to establish the major feature of the structure of magnetite [6]. The determination of the crystalline structure using X-ray diffraction is performed to obtain the crystalline lattice spacing of a sample. The crystal structure contains symmetry elements along 
planes, axes or centers. The intercept with any plane of a symmetry axis is defined by the reciprocal values of the intercepts, hkl, known as Miller indices. Magnetite and Magnetic activated carbon samples X-ray diffraction patterns are shown in Fig. 1.

X-ray powder diffraction showed that the resulting material contained nanocrystalline magnetite (see Figure 1). XRD patterns for the samples indicate that $\mathrm{Fe}_{3} \mathrm{O}_{4}$ is deposited in activated carbon. X- ray diffraction method is applied not only to structure determination, but to the measurement of particle size. The crystal size can be calculated from line broadening of the XRD pattern using the Scherrer formula shown in Equation 3. The Equation uses the corrected reference peak width at angle $\theta$, where $\lambda$ is the X-ray wavelength, B is the corrected width of the XRD peak at half height and $\mathrm{K}$ is a shape factor which is approximated by 0.89 for magnetite [7]

$$
D=\frac{0.89 \lambda}{B \cos \theta}
$$

The mean crystallite size was calculated from XRD line broadening using the Scherrer relationship. The diameters of particle of $\mathrm{Fe}_{3} \mathrm{O}_{4}$ are shown in Table 1.

TABLE 1. Particle diameter obtained from Scherrer's formula, for product samples $M_{1}$ and $M_{2}$.

\begin{tabular}{cccc}
\hline Sample & $\mathbf{B}$ (deg.) & $\boldsymbol{\theta}$ (deg.) & D (nm) \\
\hline $\mathrm{M}_{1}$ & 0.5314 & 35.5306 & 18.26 \\
$\mathrm{M}_{2}$ & 0.0787 & 35.4235 & 123.35 \\
\hline
\end{tabular}

\section{Hysteresis}

The most common way to represent the magnetic properties of a ferromagnetic material is plots of magnetization (M) against the field strengths $(\mathrm{H})$, the so-called hysteresis loop. The suitability of ferromagnetic materials for application is determined principally from characteristics shown by their hysteresis loops [8]. The magnetization properties were investigated using vibrating sample magnetometery (VSM) which provided fundamental magnetic behavior quantification. One important parameter of the magnetization properties is saturation magnetization $\left(\mathrm{M}_{\mathrm{S}}\right)$ of the particle samples. The hysteresis loop data of samples $\mathrm{M}_{1}$ and $\mathrm{M}_{2}$ particle, which was measured in the powder state at $300{ }^{\circ} \mathrm{K}$ is shown in Table2.

TABLE 2. Magnetic properties of product samples.

\begin{tabular}{ccc}
\hline Sample & $\mathbf{M}_{\mathbf{S}}(\mathbf{e m u} / \mathbf{g})$ & \% Magnetite \\
\hline $\mathrm{M}_{1}$ & 50.27 & ------ \\
$\mathrm{M}_{2}$ & 72.20 & ---- \\
$\mathrm{MAC}_{1}$ & 28.33 & 13.20 \\
$\mathrm{MAC}_{2}$ & 15.45 & 11.80 \\
\hline
\end{tabular}

The magnetization of bulk materials is inherently larger than for nanoparticles of the respective materials. Experimental values for the saturation magnetization of our samples are $50.27 \mathrm{emu} / \mathrm{g}$ for $\mathrm{M}_{1}$ and $72.20 \mathrm{emu} / \mathrm{g}$ for $\mathrm{M}_{2}$, significantly less than that of bulk magnetization, which is $92 \mathrm{emu} / \mathrm{g}$ [9]. The value of $\mathrm{M}_{\mathrm{S}}$ itself will be regarded simply as a constant of the material. Several factors affect the shape of the hystersis loop and $\mathrm{M}_{\mathrm{S}}$ value. One factor which may strongly affect is particle size. Several researchers using a variety of techniques have investigated the causes for the observed reduction in magnetization in fine magnetic particles. Results suggest that particle surface defects and orderdisorder structural characteristics both influence the magnetic properties. The values for the saturation magnetization of our samples are $28.33 \mathrm{emu} / \mathrm{g}$ for $\mathrm{MAC}_{1}$ and $15.45 \mathrm{emu} / \mathrm{g}$ for $\mathrm{MAC}_{2}$ (see Fig. 2). These are significantly less than the magnetization of the magnetic samples. This is due to strong dependence on the particle size, percent of magnetite in sample, particle surface defects and order-disorder structural dependence. 


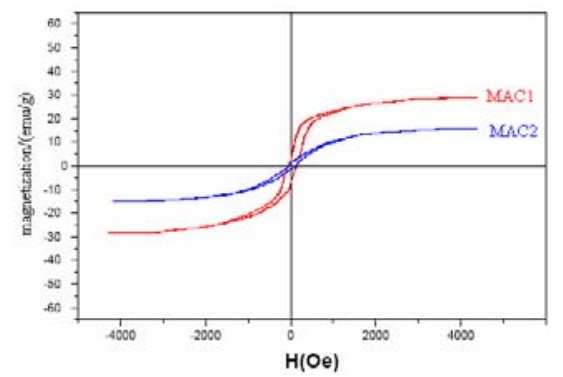

FIGURE 2. Hysteresis loop of magnetic activated carbon samples, $\mathrm{MAC}_{1}$ and $\mathrm{MAC}_{2}$.

\section{Scanning Electron Microscopy (SEM)}

The morphology and size distribution of particles are most easily obtained by SEM. SEMs showed the development of porosity of the magnetic activated carbon, and nano-crystals of inorganic magnetite deposits are clear. Figure 3 shows the $\mathrm{SEM}$ photographs of magnetite in activated carbon samples $\mathrm{MAC}_{1}$ and $\mathrm{MAC}_{2}$, respectively.

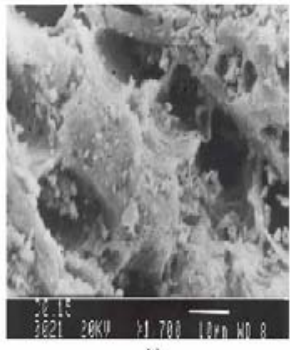

(a)

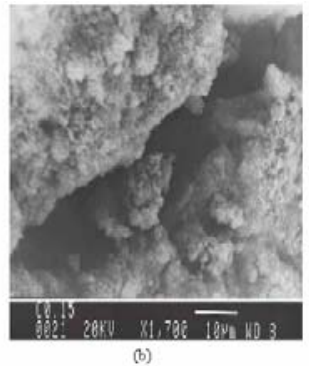

FIGURE 3. SEM photographs of magnetic activated carbon (a) $\mathrm{MAC}_{1}$, and (b) $\mathrm{MAC}_{2}$.

On the other hand, microanalysis is the determination of the composition of the specimen on a microscopic scale. In an electron microscope, this is done by irradiating with a beam of electrons and analyzing the radiation emitted. In Energy Dispersive X-Ray (EDX) qualitative identification of the elements present in a sample and their spatial distribution are possible. The minimum detection limits vary around about 0.1 percent by weight. The EDX spectra of activated carbon and magnetic activated carbon $\left(\mathrm{MAC}_{1}\right)$ are show in Fig. 4.

The results of X-ray diffraction analysis according to peak height show a strong K $\alpha$ peak for carbon and a weak $\mathrm{K} \alpha$ peak for traces of oxygen in activated carbon. In magnetic activated carbon, oxygen $\mathrm{K} \alpha$ peak increases and also $\mathrm{Fe}-\mathrm{L} \alpha$, $\mathrm{Fe}-\mathrm{K} \alpha$ and $\mathrm{Fe}-\mathrm{K} \beta$ peaks appear for magnetite deposition.

Results show that gold adsorption increases in $\mathrm{MAC}_{2}$. The percent adsorption of gold on activated carbon and homogenous magnetic activated carbon $\left(\mathrm{MAC}_{1}\right)$ and heterogeneous magnetic activated carbon $\left(\mathrm{MAC}_{2}\right)$ are $100 \%, 77 \%$, and $95 \%$, respectively. 


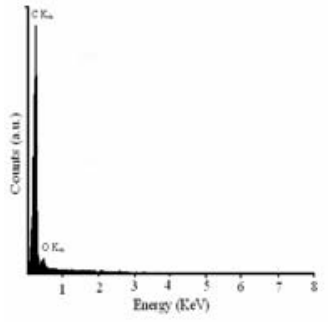

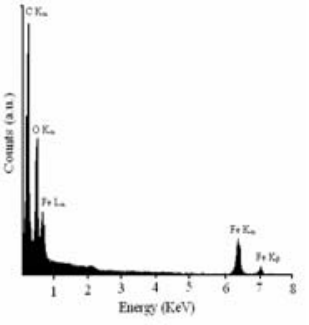

FIGURE 4. The EDX spectra of (a) activated carbon, and (b) magnetic activated carbon.

The formation of micropores and the extent of specific surface area are considered to be important factors for adsorption. According to literature, the pores of activated carbon are classified into three groups: micropores $(\mathrm{dp}<2 \mathrm{~nm})$, mesopores $(2 \mathrm{~nm}<\mathrm{dp}<50 \mathrm{~nm})$ and macropores $(\mathrm{dp}>50 \mathrm{~nm})$, where $\mathrm{dp}$ is the pore width [10]. Activation widens the micropores resulting in more developed porosity. During adsorption, macro and mesopores allow rapid transport of adsorbate into the interior of carbon, for subsequent diffusion into the micropore volume. Consequently, a welldeveloped porous network in all pore size ranges results in improved adsorption properties of the product. The porosity is dependent on the magnetic particle size on the deposit of activated carbon. In $\mathrm{MAC}_{1}$ magnetite nanoparticle can occupy mesopores and in $\mathrm{MAC}_{2}$ only deposit in macropores is possible. A better way to produce magnetic activated carbon is a heterogeneous mixture of activated carbon and the magnetic precursor.

\section{REFERENCES}

1. J. Svoboda J., Magnetic Methods for Treatment of Minerals (Elsevier, 1987).

2. E. Bernardo, R. Egashira, J. Kawasaki, Carbon 35 (1997) 1217.

3. R. C. Bansal, D. B. Donnet and F. Stoekli, Active Carbon (Marcel Dekker, New York, 1988).

4. Z. L. Liu, Y. J. Liu, K. L. Yao, Z. H. Ding, J. Tao, and X. Wang, Journal of Materials Synthesis and Processing 10 (2002) 83.

5. G. Moffat, R. A. Williams, C. Webb and R. Stirling, Miner. Eng. 7 (1994) 1039.

6. B. D. Cullity, Element of X-ray Diffraction (Addison Wesley, Reading, MA 1974).

7. H. P. Klug and L. E. Alexander, X-ray Diffraction Procedures for Polycrystalline and Amorphous Materials (J. Wiley and Sons, New York, 1974) p. 966

8. D. Jiles, Introduction to Magnetism and Magnetic Materials (Chapman and Hall, 1991) p. 71

9. M. P. Morales, M. Andres-Verges, S. Veintemillas-Verdaguer, M. I. Montero, C. J. Serna, J. Magn. Magn. Mater. 203 (1999) 146.

10. P. Galiatsatou, M. Metaxas and V. K. Rigopoulou, Mikrochimica Acta 136 (2001) 147 